International Journal of Advanced Research in Engineering and Technology (IJARET)

Volume 10, Issue 4, July-August 2019, pp. 123-127, Article ID: IJARET_10_04_014

Available online at http://iaeme.com/Home/issue/IJARET?Volume $=10 \& I s s u e=4$

ISSN Print: 0976-6480 and ISSN Online: 0976-6499

(C) IAEME Publication

\title{
HUMAN FACE DETECTION WITH SKIN COLOR PROPERTIES
}

\author{
Samira Abdul-Kader Hussain \\ Computer Science Dept./ College of Science / Mustansiriyha University, Iraq \\ Dr. Maha A. Al-Bayati \\ Computer Science Dept./ College of Science / Mustansiriyha University, Iraq
}

\begin{abstract}
It is well known that color of skin contributes heavily, as a vital feature, to detection, recognition, as well as tracking of face and hand in video applications for human. This research presents the attempts to use skin color in the process of face detection in colored image. Skin detection is considered as priori. It mainly relies on H channel in HSV color models to characterize the skin colors range. The work presented prove applicability for different input human color images of different type and size.
\end{abstract}

Key words: Face Detection, skin, color and HSV

Cite this Article: Samira Abdul-Kader Hussain and Dr. Maha A. Al-Bayati, Human Face Detection with Skin Color Properties, International Journal of Advanced Research in Engineering and Technology, 10(4), 2019, pp. 123-127.

http://iaeme.com/Home/issue/IJARET?Volume=10\&Issue $=4$

\section{INTRODUCTION}

Face detection and tracking operations of digital images has become colored proposed decades ago, that a lot of pattern recognition processes proposed extensive and accurate techniques in face detection. Of the most important methods used in face detection is to rely on skin color, as the color of the skin allows fast processing in face detection operations.

Many techniques exist for detection of face and skin [10], amongst are the Gaussian Mixture technique, thresholding, neural nets, and template matching and color histogram. A method for skin color segmentation was presented by Prashanth Kumar and Shashidhara [8] . This method uses RGB, YCbCr, and HSV color models together with thresholds to help removing non skin pixels from input image. V. Oliveira, A. Conci [5] brought up another approach for detecting skin color. This approach relies on HSV color models to implement skin detection with the aid of using the $\mathrm{H}$ channel to characterize the skin colors range. Zaher $\mathrm{H}$. Al-Tairi et al. [9] proposed another method that combines RGB with YUV spaces of color to facilitate extracting regions of colors in face skin based on thresholding technique that is liable 
to changes in lighting while gaining ability to distinguish color pixels in skin. Another method described by Ghotkar and Kharate [7] was that concerning hand segmentation. This method uses a threshold technique for hand gesture recognition, where a comparison is carried out between the three color spaces of HSV, HSL, and HTS, and observed that the latter gives better results than the two former ones.

The discussion just presented highlights the idea that, with the aid of certain threshold, the use of more than one color space is a considerable tool for effective skin color segmentation.

The color of human skin contains a range of hues and it is not saturated in deep, and that is why human skin color does not fall in a given color models. In fact it is clustered at a small object in the color models, even is not the same for all those models. So, this work attempts to recruit the skin color for face detection process in such a way to consider $\mathrm{H}$ color value after conversion of RGB color images into HSV color model, then face is detected considering the skin color and sample geometric operations.

\section{SKIN COLOR AND COLOR MODELS}

Various types of color models exist for skin detection. The RGB color model is the most commonly used model in each image format. Other color models include $\mathrm{HSV}, \mathrm{YCbCr}$, or YIQ. Even though an input image is generally in RGB format, skin color is still be part of this model, which may be built in a large area of this color model. Usually this area is called the cluster of skin color.

Classifying skin color is the first step in algorithms used for facial expression extraction, face and hand tracking and many other the classification and recognition systems. In which case, pixels are classified as a particular pixel on the skin or non-skin, depending on the color clustering in the color model.

\section{FACE DETECTION APPROACH}

The proposed Face detection approach has two major algorithms:

- Skin Color Detection Algorithm

- Face Detection Algorithm

\subsection{Skin Color Detection Algorithm}

The proposed skin color has three major components:

- Color Convert and get $\mathrm{H}$ values for each pixel; In this step, convert RGB color for each pixel into HSV color

- Classify $\mathrm{H}$ value as skin or non-skin; where each pixel is classified as being skin or non-skin using thresholding technique, that is, certain threshold values are used to distinguish the skin regions.

- Enhancement Binary image result. 


\title{
Algorithm (1) : Skin Detection
}

\author{
Step1: Start \\ Step2: Read a color image personal \\ Step3: Convert color data from RGB to HSV color model \\ Step4: Calculate $\mathbf{H}$ value by:

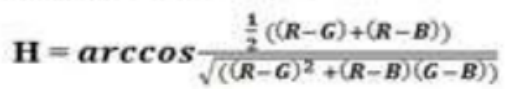 \\ Step5: If $0 \leq \mathrm{H} \geq 50$ then $\mathrm{H}$ pixel-value $=1$, \\ Else $\mathrm{H}$ pixel-value $=\mathbf{0}$ \\ Step6: Apply Median filter on result image (step 5) \\ Step 7: Skin detect for white pixel result \\ Step8: End
}

\subsection{Face Detection Algorithm}

A region $\mathrm{R}$ (in a binary image) has a boundary which is the set of all pixels in $\mathrm{R}$ that have at least one neighbor out of $\mathrm{R}$. It happens that $\mathrm{R}$ can be an entire image (rectangular) rows and columns of the image. In this sense, referring to a region means referring to a subset(i.e. part) of an image, and any number of pixels in the boundary of a region, that happens to coincide with the border of the image, would be implicitly included as part of that region's boundary. From this perspective, the face is the greatest part of the image in personal face images,

\section{Algorithm (2) : Face Detection}

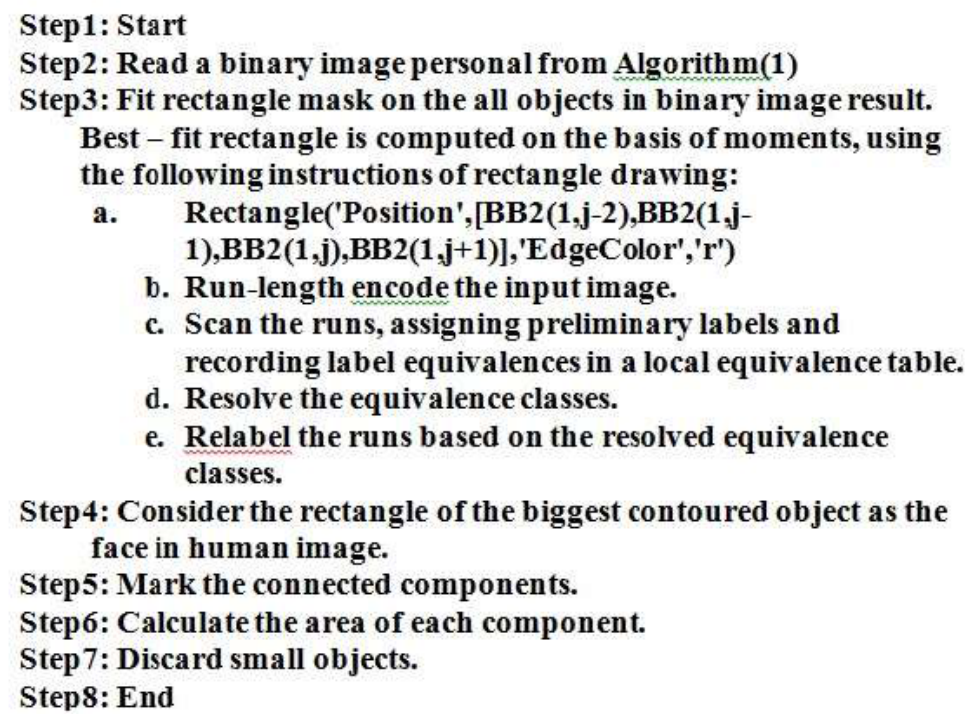

\section{EXPERIMENTAL RESULTS}

Being the core step in this work, skin detection is used to accomplish the task to identifying human face in input personal images. Among a total of 30 images, 20 images are used for training, and the rest 10 images are used for testing. 
All experiments are held under conditions that all of input images are of the same size, and that only frontal view of human face in an image is to be analyzed during the course of work in the system.

The result of applying skin detection algorithm to a sample test face image, namely Face1, is presented in figure(1), where (a) is the original test image, (b) shows HSV color after converting RGB color to HSV color, and (c) shows the Black/ White color considering H value.

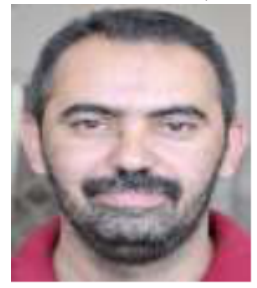

(a)

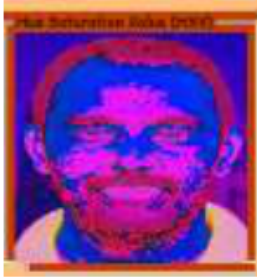

(b)

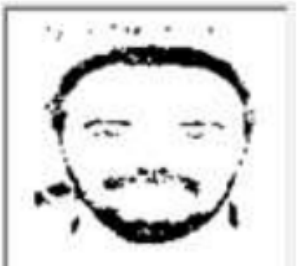

(c)

Figure 1 Skin detection for Face1: (a) Original test image, (b) HSV color $\quad$ (c) B/W color.

Figure (2) shows the result of applying face detection algorithms to another test face image, namely Face2. Again, (a) is the original test image,(b) depicts skin color resulted by applying skin detection algorithm, (c) depicts the result of localizing regions of interest (i.e. white objects surrounded by red rectangles), and (d) face detected from one of these objects (the largest amongst others).

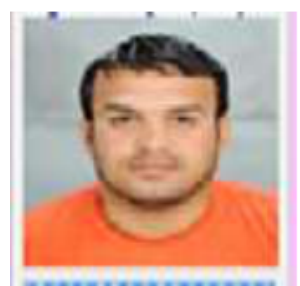

(a)

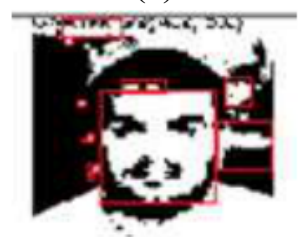

(c)

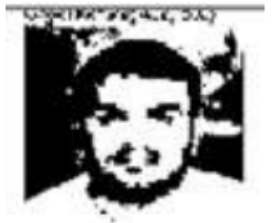

(b)

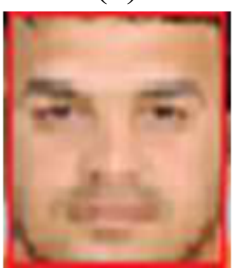

(d)

Figure 2 Face2 test image for face detection step: (a) Original test image, (b) skin color detection, (c) Localization of objects in image, (d) face detection. 


\section{CONCLUSION}

This research developed a method for detecting human faces in colored images on the bases of skin color. A number of image processing techniques was put together to achieve best results in this concern.

A face detection step was done, on principle that, the face is the biggest part constituting a person image in .jpg image file. This drove the work towards selecting the bigger object that would result from applying skin detection step to find a face in the input personal image.

\section{REFERENCES}

[1] BRAND, J. et al.," A Comparative Assessment of Three Approaches to Pixel-level Human Skin-detection", In Proceedings of the International Conference on Pattern Recognition, Vol. 1, 2000, pp. 1056-1059.

[2] Vladimir V. et al.," A Survey on Pixel-Based Skin Color Detection Techniques", Faculty of Computational Mathematics and Cybernetics, 2004.

[3] Gonzalez R. C. et al. , "Digital Image Processing", Addison-Wesley Publishing Company, 2004.

[4] Kakumanu P. et al., "A Survey of Skin-color Modeling and Detection Methods", ITRI/Department of Computer Science and Engineering, Wright State University, Dayton OH 45435, USA, 2006.

[5] Oliveira V. et al.," Skin Detection using HSV color space", In H. Pedrini, \& J. Marques de Carvalho, Workshops of Sibgrapi, 2009, pp. 1-2.

[6] Tabassum M. R. et al.," Comparative Study of Statistical Skin Detection Algorithms for Sub-Continental Human Images", Information Technology Journal Vol. 9, Issue 4, pp. 811817,2010 .

[7] Archana S. Ghotkar et al.," Hand Segmentation Techniques to Hand Gesture Recognition for Natural Human Computer Interaction", International Journal of Human Computer Interaction (IJHCI), Vol. 3, Issue 1, 2012.

[8] Prashanth Kumar G. et al.," Real Time Detection and Tracking of Human Face Using Skin Color Segmentation and Region Properties", International Journal of Signal Processing Systems Vol. 2, No. 2, December 2014

[9] Zaher Hamid Al-Tairi et al., "Skin Segmentation Using YUV and RGB Color Spaces ", J Inf Process Syst, Vol.10, No.2, June 2014, pp.283-299,

[10] Samira A. H. et al., "Eyes Detection in the Human Face", International Journal of Civil Engineering and Technology, Vol.9, Issue 10, October. 2018, pp. 1001-1007, 